\title{
Neutrino physics with the SHiP experiment
}

\author{
Annarita Buonaura*广 \\ Universitá di Napoli Federico II e INFN Napoli \\ E-mail: annarita.buonaura@na. infn. it
}

Despite the Standard Model (SM) has been strongly confirmed by the Higgs discovery, several experimental facts are still not explained. The SHiP experiment (Search for Hidden Particles), a beam dump experiment at CERN, aims at the observation of long lived particles very weakly coupled with ordinary matter. These particles of the $\mathrm{GeV}$ mass scale, foreseen in many extensions of the SM, might come from the decay of charmed hadrons produced in the collision of a $400 \mathrm{GeV}$ proton beam on a target.

High rates of all the three active neutrinos are also expected. For the first time the properties and the cross section of the $v_{\tau}$ will be studied thanks to a detector based on nuclear emulsions, with the micrometric resolution needed to identify the tau lepton produced in neutrino interactions. Measuring the charge of the tau daughters, will enable the first observation of the $\bar{v}_{\tau}$ and the study of its cross section.

XVI International Workshop on Neutrino Telescopes,

2-6 March 2015

Palazzo Franchetti - Istituto Veneto, Venice, Italy

\footnotetext{
* Speaker.

On behalf of the SHiP Collaboration.
} 


\section{Introduction and physics motivations}

In the latest decades, the Standard Model (SM) of elementary particle physics has become the most validated theory that provides a consistent description of Nature's fundamental constituents and of their interactions. Its predictions have been tested and confirmed by numerous experiments, the latest of all being the discovery of the Higgs boson in 2012 [1],[2]. So far, nor direct or indirect searches for new physics have found any evidence for significant deviations from the SM although it is clear that it is not a complete theory. Indeed it is unable to explain a certain number of observed phenomena in particle physics, astrophysics and cosmology such as neutrino masses and oscillations, baryon asymmetry of the universe (BAU) and Dark Matter (DM). The answer to these questions might come from the hypothesis that some yet unknown particles exist. They might have never been detected because too heavy to be produced at current collision energies or because too weakly interacting with ordinary SM particles.

So far, all major particle physics experiments have focused their attention to reach always increasing collision energies, thus exploring the "energy frontier". On the other hand, the SHiP experiment (Search for Hidden Particles, [3], [4]) is a beam dump facility, intended to operate at CERN, designed to explore the complementary path to these searches by moving towards much lower interaction strengths ("intensity frontier"). It will explore the so called Hidden Sector, looking for: new particles which are singlets with respect to the SM gauge group, light sgoldstinos that appear in the breaking of symmetry of the SUSY theory, sterile neutrinos called Heavy Neutral Leptons (HNL) also foreseen in a minimal extension ( $v$ MSM [5]) of the SM as heavy (order of GeV) right handed partners of the SM neutrinos to explain DM, BAU as well as the origin of neutrino masses. With a proton energy enhancing the production of charmed mesons and in particular of $D_{s}$ mesons, a high flux of $v_{\tau}$ and $\bar{v}_{\tau}$, together with neutrinos of all flavours, is expected. Therefore, the facility will host a tau neutrino detector to observe the tau anti-neutrino (only missing tile in the SM), study $v_{\tau}$ s properties and cross-section and neutrino induced charm production by $v_{e}$ and $v_{\mu}$.

\section{The SHiP facility}

The overall detector layout is shown in fig.1. Protons accelerated at the SPS up to $400 \mathrm{GeV} / \mathrm{c}$, with an integrated intensity of $2.0 \times 10^{20}$ protons on target (pot) along five years of run, hit a hybrid target (TZM molybdenum alloy and pure tungsten). At these energies Hidden Particles (HP) in the $\mathrm{GeV}$ range might be produced in the decay of charm and beauty hadrons, thus making it necessary to enhance the production of these hadrons.

To detect HP, the idea is to reconstruct their exclusive decays in SM particles. The smallness of the couplings implies that HP have typical lifetimes longer than $10 \mu \mathrm{s}$ and a decay distance of $\mathrm{O}(\mathrm{km})$. Being produced in charmed hadrons decays, HP also show significant transverse momentum, hence requiring, to maximize the acceptance, to place the decay volume, consisting of an evacuated $50 \mathrm{~m}$ long tube with a diameter of $5 \mathrm{~m}$, as close as possible to the proton target. To fully reconstruct and perform the identification of particles produced in HP decays, magnetic spectrometer, calorimeters and muon detectors are placed at the far end of the decay volume.

The facility is also designed to minimize background sources such as: the muon flux coming from short-lived resonances and from the residual flux of decaying pions and kaons, the active neutrino 


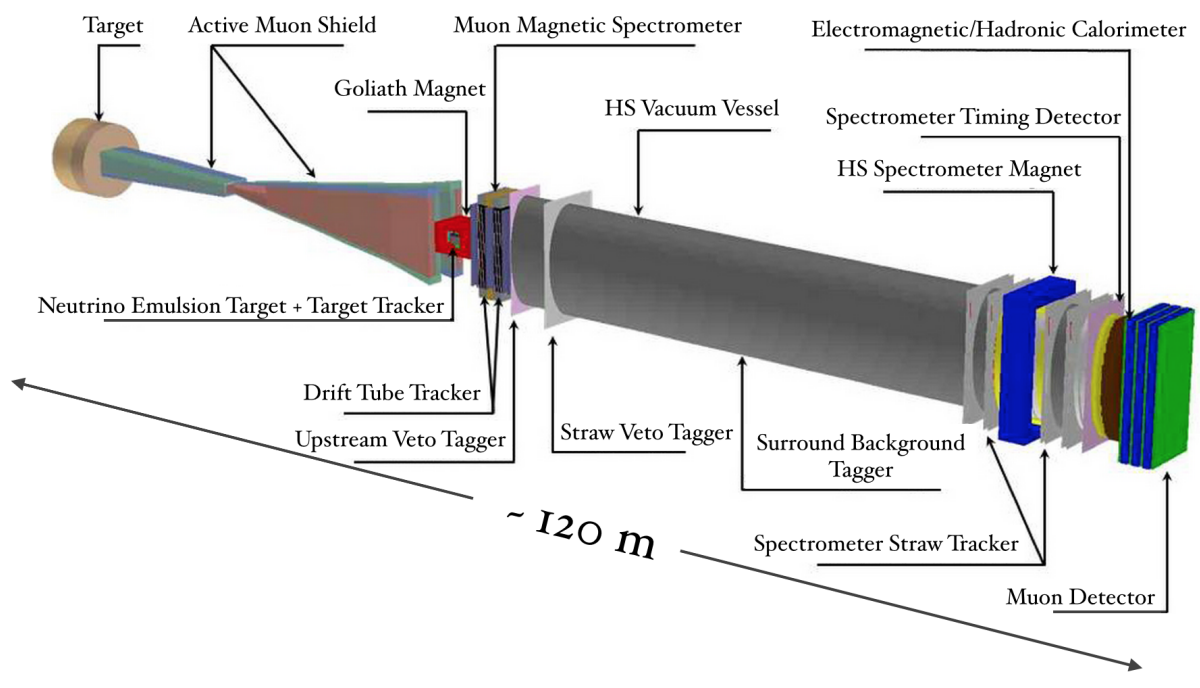

Figure 1: Overall detector layout of the SHiP facility.

flux. A $48 \mathrm{~m}$ long active muon shield based on magnetic deflection of the muons in the horizontal plane clears up a $5 \mathrm{~m}$ horizontally wide region from muons. The combinatorial background from the residual muons entering the decay volume and muons scattered off the cavern walls is reduced by proton spills with a slow (1s) and very uniform beam extraction. Neutrino interactions inside the decay volume are negligible if the vessel is kept at low pressure $\left(10^{-6} \div 10^{-5} \mathrm{bar}\right)$, while to provide efficient protection against neutral particles produced in charged-current (CC) and neutral-current (NC) neutrino interactions in the material upstream of the fiducial volume, a combination of light taggers is located upstream and at the beginning of the fiducial volume.

\subsection{The neutrino detector}

A neutrino detector (fig.2a) is designed to perform the first direct observation of the $\bar{v}_{\tau}$ and to study the properties and the cross-section of $v_{\tau}$ and $\bar{v}_{\tau}$. It is located immediately downstream of the active muon shield and it is a modular target. The fundamental unit is made of a brick and a Compact Emulsion Spectrometer (CES) as shown in fig.2b. The brick employs the Emulsion Cloud Chamber (ECC) technology interleaving lead plates serving as passive material and emulsion films serving as tracking devices with micrometric resolution. This technology allows to identify the tau neutrino by disentangling the $\tau$ lepton production and decay vertices, as previously done in the 4 $v_{\tau}$ candidate OPERA [6] events [7], [8], [9], [10].

The measurement of the electric charge of the $\tau$ daughters for the $v_{\tau}$ and anti- $v_{\tau}$ identification is performed by a CES attached downstream of each ECC brick. It is made of a sandwich of light material plates (Rohacell) and emulsion films for a total length of $3.1 \mathrm{~cm}$ and it is designed in order to be able to perform charge measurement for hadrons with $\mathrm{O}(\mathrm{GeV})$ momentum, being the target placed in a magnetic field of about $1 \mathrm{~T}$.

The complete target consists of more than one thousand units, for a total mass of about 9.6 tons. It is also complemented by planes of electronic detectors to provide the time stamp of the event, to link the muon tracks in the target with the magnetic spectrometer.

As a matter of fact, muon identification at neutrino interaction vertex is crucial since that the main 


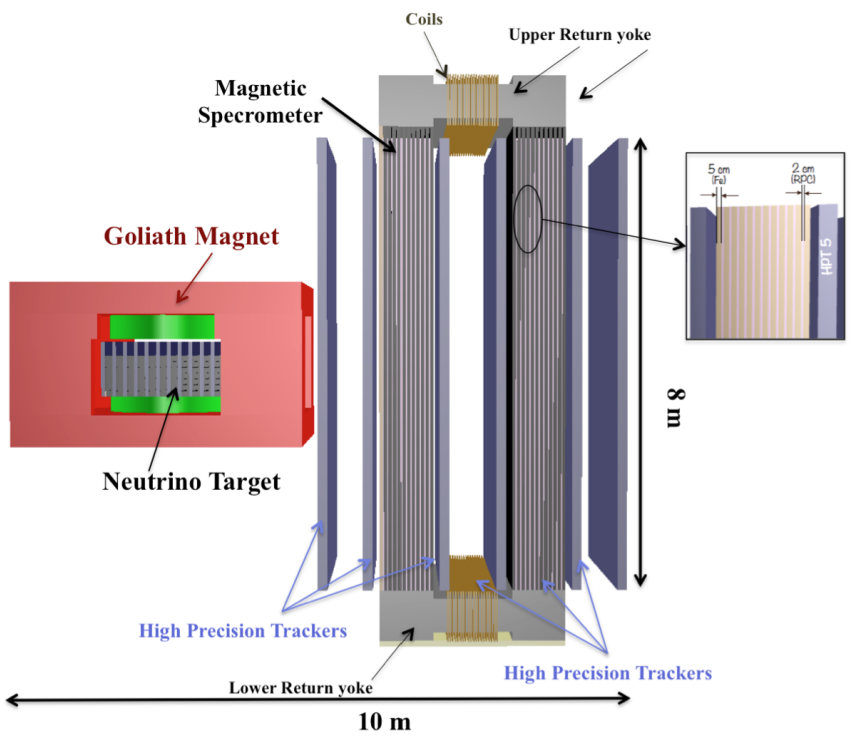

(a) Layout of the neutrino detector.

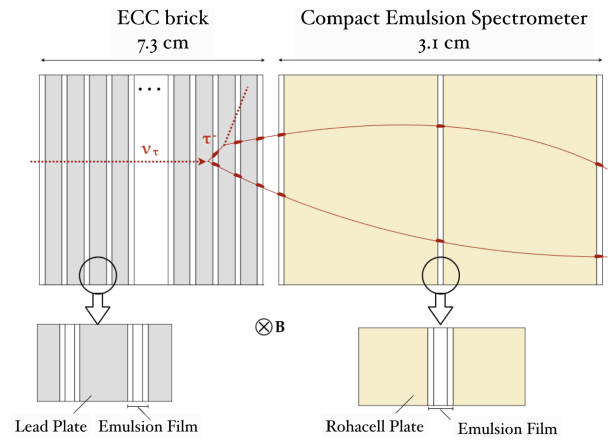

(b) Schematic representation of the neutrino detector unitary cell.

Figure 2: View of the tau neutrino detector and of its fundamental unit.

background for the $v_{\tau}$ search comes from charmed hadrons produced in $v_{\mu}$ CC interactions where the primary lepton is not identified.

\section{Physics with $v_{\tau}$}

Tau neutrinos are copiously produced via $D_{s} \rightarrow \tau \nu_{\tau}$ and subsequent $\tau \rightarrow \nu_{\tau}$ decay. The number of estimated $v_{\tau}+\bar{v}_{\tau}$ produced is of $5.7 \times 10^{15}$ per $2 \times 10^{20}$ protons on target. Electron and muon neutrinos are also induced by the decay of soft pions and kaons produced as secondary particles in proton interactions. A Geant 4 based simulation of the target and of the hadron stopper provides the spectra shown in fig. 3a and the yield reported in the left colum of tab. 1. The expected number of charged-current deep-inelastic neutrino interactions in a 9.6 ton detector in five years run is reported in the right column of Tab. 1 while the energy spectra are shown in fig. 3c. It is important to notice that almost $7000 v_{\tau}$ and $3500 \bar{v}_{\tau}$ charged current interactions are expected.

This unprecedented statistics of tau neutrinos and anti-neutrinos will allow to perform cross section studies and it will enable the extraction of the $F_{4}$ and $F_{5}$ structure functions participating to the charged-current $v_{\tau}\left(\bar{v}_{\tau}\right)$ deep inelastic differential cross-section. As a matter of fact, $F_{4}$ and $F_{5}$ are negligible in muon (and electron) neutrino interactions because of a suppression factor proportional to the square of the charged lepton mass. At leading order, in the limit of massless quarks and target hadrons, $F_{4}=0$ and $2 x F_{5}=F_{2}$, where $x$ is the Bjorken- $x$ variable [11]. Calculations at NLO show that $F_{4}$ is about $1 \%$ of $F_{5}$ [12].

Other measurements will improve the current knowledge of fundamental quantities: the strangequark content of the nucleon can be measured by means of the charmed hadron production in anti-neutrino interactions. In 5 years run, more than $10^{5}$ neutrino induced charmed hadrons are expected, exceeding the statistics available in previous experiments by more than one order of 


\begin{tabular}{c|cc|cc}
\hline & $<$ E $>$ & Beam dump & $<$ E $>$ & CC DIS \\
\hline$N_{v_{\mu}}$ & 1.4 & $4.4 \times 10^{18}$ & 29 & $1.7 \times 10^{6}$ \\
$N_{v_{e}}$ & 3 & $2.1 \times 10^{17}$ & 46 & $2.5 \times 10^{5}$ \\
$N_{v_{\tau}}$ & 9 & $2.8 \times 10^{15}$ & 59 & $6.7 \times 10^{3}$ \\
$N_{\bar{v}_{\mu}}$ & 1.5 & $2.8 \times 10^{18}$ & 28 & $6.7 \times 10^{5}$ \\
$N_{\bar{v}_{e}}$ & 4 & $1.6 \times 10^{17}$ & 46 & $9.0 \times 10^{4}$ \\
$N_{\bar{v}_{\tau}}$ & 8 & $2.8 \times 10^{15}$ & 58 & $3.4 \times 10^{3}$ \\
\hline
\end{tabular}

Table 1: Integrated neutrino yield for $2 \times 10^{20}$ p.o.t. for the different neutrino flavors: at the beam dump (left) and CC DIS interactions (right). Energies are in $\mathrm{GeV}$.

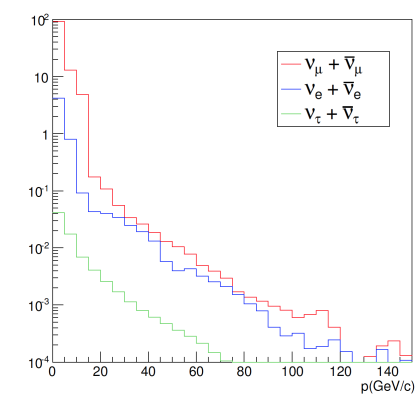

(a) Beam dump.

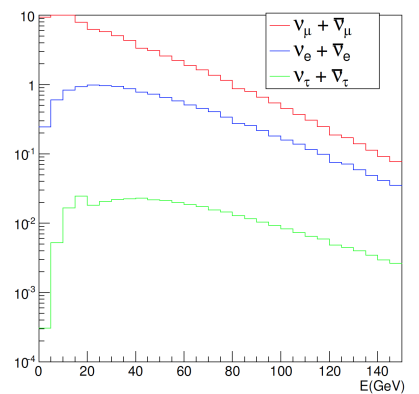

(b) CC interacting.

Figure 3: Energy spectra of all neutrino flavors at the beam dump (a) and giving $\mathrm{CC}$ interactions in the neutrino target(c). The total number of neutrinos is normalized to 100.

magnitude. A simulated SHiP data sample normalized to the expected statistics was used to estimate the distribution of $v_{\mu}\left(\bar{v}_{\mu}\right)$ interactions with charm production. The potential impact of simulated charm data was assessed by adding them to the NNPDF3.0 NNLO fit [13]. Defining $s^{ \pm}$ as $s^{ \pm}=s(x) \pm \bar{s}(x)$, almost a factor two gain on $s^{+}$is achieved in the $x$ range between 0.03 and 0.35 and in the one between 0.08 and 0.30 for $s^{-}$.

\section{References}

[1] ATLAS Collaboration, Phys.Lett. B716 (2012) 129.

[2] CMS Collaboration, Phys.Lett. B716 (2012) 3061.

[3] SHIP Collaboration, CERN-SPSC-2015-016, SPSC-P-350, arXiv:1504.04956 [physics.ins-det], (2015)

[4] SHIP Collaboration, CERN-SPSC-2015-017, arXiv:1504.04855 [hep-ph], (2015).

[5] T.Asaka, M.Shaposhnikov, PL B620 (2005) 17.

[6] OPERA Collaboration, JINST 4 (2009) P04018.

[7] OPERA Collaboration, Phys.Lett. B691 (2010) 138-145.

[8] OPERA Collaboration, JHEP 11 (2013) 036.

[9] OPERA Collaboration, Phys. Rev. D89 (2014) 051102.

[10] OPERA Collaboration, PTEP. 2014 (2014) $10101 \mathrm{C} 01$.

[11] C. H. Albright and C. Jarlskog, Nucl. Phys. B 84 (1975) 467.

[12] M. H. Reno, Phys. Rev. D 74 (2006) 033001.

[13] R. D. Ball et al., Parton distributions for the LHC Run II. (2014). 